non-smoking woman with a positive antisynthetase antibody and otherwise unexplained NSIP and organising pneumonia may have a different ILD phenotype, prognosis and response to treatment than an older Caucasian cigarette smoking man with a positive rheumatoid factor and UIP on surgical lung biopsy who meets IPAF criteria through either a diffuse lymphoplasmacytic infiltrate or unexplained multi-compartment involvement of the airways, pulmonary vasculature or pleural or pericardial abnormalities. In addition, as many patients with NSIP and organising pneumonia are treated with immunosuppressive therapy if there is any suggestion of an autoimmune phenotype, the performance of the IPAF criteria in patients with UIP and unclassifiable ILD may be most important of all [6].

In summary, we agree with A.S. Jee and colleagues that evaluation of the patient with interstitial pneumonitis requires a multidisciplinary collaboration including rheumatology, with uniformity and standardisation in CTD definitions when applying the IPAF criteria. We are excited and inspired by the recognition, research and dialogue the formulation and publication of the IPAF criteria has generated. We look forward to the day when all patients will have a validated assessment of the contribution of autoimmunity to their ILD with a resulting diagnosis that accurately reflects their prognosis and response to treatment.

@ERSpublications

IPAF criteria need to be implemented in a standard fashion across centres for accurate validation http://ow.ly/W7Fz304BKq0

Mary E. Strek ${ }^{1}$, Justin M. Oldham ${ }^{2}$, Ayodeji Adegunsoye ${ }^{1}$ and Rekha Vij ${ }^{1}$

${ }^{1}$ Dept of Medicine, Section of Pulmonary and Critical Care Medicine, The University of Chicago, Sacramento, CA, USA. ${ }^{2}$ Dept of Medicine, The University of California at Davis, Sacramento, CA, USA.

Correspondence: Mary E. Strek, Dept of Medicine, The University of Chicago, 5841 S. Maryland Ave, MC6076 Chicago, IL 60637, USA. E-mail: mstrek@medicine.bsd.uchicago.edu

Received: Sept 192016 | Accepted: Sept 202016

Conflict of interest: Disclosures can be found alongside this article at erj.ersjournals.com

\title{
References
}

1 Oldham JM, Adegunsoye A, Valenzi E, et al. Characterisation of patients with interstitial pneumonia with autoimmune features. Eur Respir J 2016; 47: 1767-1775.

2 Fischer A, Antoniou KM, Brown KK, et al. An official European Respiratory Society/American Thoracic Society research statement: interstitial pneumonia with autoimmune features. Eur Respir J 2015; 46: 976-987.

3 Morisset J, Johnson C, Rich E, et al. Management of myositis related interstitial lung disease. Chest 2016; [in press DOI: 10.1016/j.chest.2016.04.007].

4 Witt LJ, Curran JJ, Strek ME. The diagnosis and treatment of the antisynthetase syndrome. Clin Pulm Med 2016; 23: 218-226.

5 Luppi F, Wells AU. Interstitial pneumonia with autoimmune features (IPAF): a work in progress. Eur Respir J 2016; 47: $1622-1624$.

6 Collins B, Raghu G. Interstitial pneumonia with autoimmune features: the new consensus-based definition for this cohort of patients should be broadened. Eur Respir J 2016; 47: 1293-1295.

\section{Smoke-free legislation and its impact on paediatric respiratory health}

To the Editor:

We read with great interest the study by VARDAVAS et al. [1] confirming the independent and interacting roles of tobacco smoke exposure during pregnancy and after birth in the development of respiratory diseases.

In the accompanying editorial, ZAIRINA [2] therefore appropriately calls for the protection of pregnant women and young children from tobacco smoke exposure. In so doing, she highlighted a number of potentially effective tobacco control interventions. We were, however, surprised to see that perhaps the most obvious public health intervention to protect the population from tobacco smoke was not mentioned: the implementation of smoke-free legislation. A large body of evidence now clearly shows that implementation 
of comprehensive smoke-free legislation is associated with improvements in the respiratory health of both children and adults [3,4]. For example, this evidence has demonstrated reductions in the risk of hospitalisations from asthma exacerbations [5], and the potential to reduce hospitalisations from acute respiratory tract infections [6-8]. Reductions in second-hand smoke exposure in public places as well as the home environment are likely to have been responsible for these improvements [9].

Smoke-free legislation is probably the most straightforward population-wide policy intervention to protect people from tobacco smoke exposure, and its health impact is well supported by a large body of existing evidence [3-5]. As ZAIRINA [2] appropriately points out, the lack of studies assessing the effectiveness of tobacco control policies in low- and middle-income countries is a particularly important knowledge gap which needs to be addressed and this also accounts for smoke-free legislation [2,5]. Over $80 \%$ of the world's population is currently not covered by comprehensive smoke-free laws [10]. Therefore, we argue that alongside the suggested focus on developing new interventions, it is important to globally increase the uptake of such policies whose effectiveness has already been established to protect the health of both children and adults.

0 @ERSpublications

Protecting children from tobacco smoke exposure via smoke-free legislation can benefit respiratory health http://ow.ly/FlHe3033Xr9

Timor Faber ${ }^{1,2}$, Aziz Sheikh ${ }^{3,4,5,6}$ and Jasper V. Been ${ }^{1,3,4}$

${ }^{1}$ Division of Neonatology, Dept of Paediatrics, Erasmus University Medical Centre - Sophia Children's Hospital, Rotterdam, The Netherlands. ${ }^{2}$ Dept of Public Health, Erasmus University Medical Centre, Rotterdam, The Netherlands. ${ }^{3}$ School for Public Health and Primary Care (CAPHRI), Maastricht University Medical Centre, Maastricht, The Netherlands. ${ }^{4}$ Centre of Medical Informatics, Usher Institute of Population Health Sciences and Informatics, University of Edinburgh, Edinburgh, UK. ${ }^{5}$ Division of General Internal Medicine and Primary Care, Brigham and Women's Hospital/Harvard Medical School, Boston, MA, USA. ${ }^{6}$ Dept of Medicine, Harvard Medical School, Boston, MA, USA.

Correspondence: Jasper V. Been, Division of Neonatology, Erasmus University Medical Centre - Sophia Children's Hospital, PO Box 2060, 3000 CB Rotterdam, The Netherlands. E-mail: j.been@erasmusmc.nl

Received: July 152016 | Accepted: Aug 032016

Support statement: J.V. Been was funded by the Erasmus University Medical Centre (Rotterdam, the Netherlands) and the Netherlands Lung Foundation (Amersfoort, the Netherlands) (grant no. 4.2.14.063JO). Funding information for this article has been deposited with Open Funder Registry.

Conflict of interest: None declared.

\section{References}

1 Vardavas CI, Hohmann C, Patelarou E, et al. The independent role of prenatal and postnatal exposure to active and passive smoking on the development of early wheeze in children. Eur Respir J 2016; 48: 115-124.

2 Zairina E. Maternal passive smoking and the risk of developing wheeze in children: how should we deal with it? Eur Respir J 2016; 48: 3-5.

3 Tan CE, Glantz SA. Association between smoke-free legislation and hospitalizations for cardiac, cerebrovascular, and respiratory diseases: a meta-analysis. Circulation 2012; 126: 2177-2183.

$4 \quad$ Kuehni CE, Barben J. Protecting children from second-hand smoke. Eur Respir J 2015; 46: 601-603.

5 Been JV, Nurmatov UB, Cox B, et al. Effect of smoke-free legislation on perinatal and child health: a systematic review and meta-analysis. Lancet 2014; 383: 1549-1560.

6 Been JV, Millett C, Lee JT, et al. Smoke-free legislation and childhood hospitalisations for respiratory tract infections. Eur Respir J 2015; 46: 697-706.

7 Lee SL, Wong WH, Lau YL. Smoke-free legislation reduces hospital admissions for childhood lower respiratory tract infection. Tob Control 2016 [in press DOI: 10.1136/tobaccocontrol-2015-052541]

8 Hawkins SS, Hristakeva S, Gottlieb M, et al. Reduction in emergency department visits for children's asthma, ear infections, and respiratory infections after the introduction of state smoke-free legislation. Prev Med 2016; 89: $278-285$.

9 Hoffman SJ, Tan C. Overview of systematic reviews on the health-related effects of government tobacco control policies. BMC Public Health 2015; 15: 744.

10 World Health Organization. WHO report on the global tobacco epidemic, 2015. Raising taxes on tobacco. WHO, Geneva, 2015. 